\title{
Chronique bibliographique. Europe, frontières et politique : chronique autour d'Etienne Balibar
}

\author{
Julien Jeandesboz
}

\section{(2) OpenEdition \\ 1 Journals}

\section{Édition électronique}

URL : http://journals.openedition.org/conflits/12963

DOI : $10.4000 /$ conflits. 12963

ISSN : $1777-5345$

Éditeur :

CCLS - Centre d'études sur les conflits lilberté et sécurité, L'Harmattan

\section{Édition imprimée}

Date de publication : 20 juillet 2008

Pagination : 191-195

ISBN : 978-2-296-05909-2

ISSN : 1157-996X

\section{Référence électronique}

Julien Jeandesboz, «Chronique bibliographique. Europe, frontières et politique : chronique autour d'Etienne Balibar », Cultures \& Conflits [En ligne], 70 | été 2008, mis en ligne le 03 octobre 2008, consulté le 30 mars 2021. URL : http://journals.openedition.org/conflits/12963 ; DOI : https://doi.org/ 10.4000/conflits.12963 


\title{
Chronique bibliographique. Europe, frontières et politique : chronique autour d'Etienne Balibar
}

\author{
Julien JEANDESBOZ
}

Julien Jeandesboz est doctorant en sciences politiques et relations internationales à l'Institut d'études politiques de Paris, et chercheur associé au centre d'études sur les conflits. Il est membre $d u$ c.a.s.e. collective.

T a récente publication d'une " petite conférence sur la frontière ${ }^{1}$ », initialement Lprononcée le 18 novembre 2006 au Centre dramatique national de Montreuil, constitue un excellent prétexte pour revenir sur les réflexions menées par Etienne Balibar depuis plus de dix ans sur l'Europe, ses frontières et ses citoyens ${ }^{2}$. La parution de cette "petite conférence », donnée devant un public majoritairement constitué de jeunes auditeurs, illustre à elle seule certaines des préoccupations de l'auteur quant au rôle général du travail (des) intellectuel(s) et quant au traitement d'une question qui, loin de n'être qu'un thème à laisser aux experts et aux militants, concerne également les sociétés et les citoyens européens dans leur ensemble. Son titre, Très loin et tout près, résume de manière simple l'une des préoccupations de la réflexion proposée par Balibar sur l'Europe, à savoir la nécessité de problématiser la constitution (à tous les sens du terme) d'un espace de gouvernement européen et les limites qui lui sont données sur le plan interne comme sur le plan externe par les acteurs dominants des processus d'européanisation communautaires, sous un angle politique qui serait celui d'un «droit de cité » conjoint aux ressortissants communautaires et extra-communautaires.

Il s'agit évidemment de resituer les propositions de Balibar dans leur contexte : une préoccupation, d'abord, pour des processus plus spécifiquement européens,

1. Balibar E., Très loin et tout près : petite conférence sur la frontière, Paris, Bayard, 2007.

2 . Cette chronique s'intéresse en particulier aux ouvrages suivants (dans l'ordre chronologique de première publication) : Balibar E., Droit de cité, Paris, Presses universitaires de France, 2002 (1 ${ }^{\text {re }}$ publication 1998); Balibar E., Nous, citoyens d'Europe? : les frontières, l'Etat, le peuple, Paris, La Découverte, 2001 ; Balibar E., L'Europe, l'Amérique, la guerre : réflexions sur la médiation européenne, Paris, La Découverte, 2005 (1 ${ }^{\mathrm{re}}$ publication 2003); Balibar E., Europe Constitution Frontière, Bègles, Editions du Passant, 2005. 
liés à la condition des sans-papiers en France, à la montée électorale des formations d'extrême droite dans les pays membres de l'UE, ou encore à la formalisation d'un processus constitutionnalisant communautaire par le biais de la convention sur le futur de l'Europe ; et une préoccupation conjointe, pour les rapports entre les espaces de l'européanisation communautaire et les espaces des relations internationales, dans le contexte des guerres de démantèlement de la fédération yougoslave, et de l'invasion de l'Irak par la coalition américano-britannique. Ces éléments servent d'appui à une réflexion visant à associer systématiquement ce que nous appellerons, dans une distinction sans doute un peu schématique, les processus politiques internes et externes aux espaces de l'européanisation communautaire.

C'est dans cette association, placée sous l'angle d'une réflexion politique, que se situent sans doute les éléments les plus stimulants des propositions d'Etienne Balibar et qu'émerge son intérêt appuyé pour les questions de frontières. Réflexion politique dans le sens où elle interpelle les processus d'européanisation sous l'angle du vivre-ensemble et de la possibilité de faire les faire correspondre "à une progression historique de la citoyenneté démocratique, et non à un détournement d'objet et d'attention ${ }^{3}$ ", par le biais notamment de "l'idée d'un "droit de cité européen" entendu comme "un droit de cité en Europe", c'està-dire une expansion de la démocratie par le moyen de la construction européenne 4 ». Il s'agit en particulier de se préoccuper de ces « exilés », réfugiés, demandeurs d'asile ou « sans-papiers ", qui cristallisent, au travers de leurs situations, les contradictions profondes d'un processus de construction communautaire qui semble s'orienter, par le biais des politiques d'immigration, de frontières et de visas communautarisées, non pas vers l'émergence d'un demos débarrassé des ethnos des Etats nationaux, mais bien vers la reconduction des pratiques d'exclusion inhérentes à ces derniers, à l'échelle de l'UE. A ce titre, rappeler que «[c]omme Européens présents et à venir, nous ne nous trouvons plus simplement situés an bord, on de part et d'autre d'une "superfrontière" géo-politique [...] mais, de plus en plus, au milieu d'une zone-frontière aux multiples dimensions ${ }^{5}$ " devient, plus qu'un jeu de métaphores, une pratique salutaire.

Face à l'institution officielle d'une identité européenne qui semble d'une part se satisfaire de quelques formalismes (un drapeau, un hymne, une élection, une constitution précautionneusement qualifiée de « traité » et, dans tous les cas, rapidement évacuée) et, d'autre part, reconduire des logiques classiques d'opposition et d'inimitié (qu'il s'agisse alors des Etats-Unis ou du radical islamique soupçonné de terrorisme), il s'agit de souligner que ces frontières que les sites étatiques européens confèrent à l'Europe constituent autant de lignes de faille, de fracture mais aussi de fuite qui traversent les espaces de l'européanisation communautaire.

3. Balibar E., Nous citoyens d'Europe ?, op. cit., p. 13. Soulignement d'origine.

4. Ibid., p. 25.

5. Balibar E., Europe Constitution Frontière, op. cit., p. 95. 
Cette logique conduit Balibar à ouvrir, de façon simultanée, une série de thèmes jouant sur le lien, qu'il considère inextricable, entre processus de constitutionnalisation et processus de «frontiérisation » européens. Les processus de constitutionnalisation renvoient ainsi à "une problématique de la "frontière intérieure" issue $d u$ dépassement des identités nationales antagonistes ", correspondant à une tentative pour "retourner leur usage étatique traditionnel pour en faire des instruments de communication plutôt que d'exclusion ${ }^{6}$. Il existe toutefois une contradiction entre les exigences formelles et matérielles de ces processus : entre les termes du débat constitutionnel mené par les gouvernants européens - illustré par le contenu des travaux de la convention constitutionnelle, mais également par le traité de Lisbonne, «simplifié » voire simpliste - et ses « apories 7 », et ce que Balibar nomme «les urgences de la conscience sociale 8 » qui renvoient entre autres à la possibilité, pour les peuples européens, de revendiquer l' «Europe » comme terme et espace de revendication et de luttes, voire de désobéissance ${ }^{9}$.

Compris comme processus de «frontiérisation interne », les processus de constitutionnalisation européens ne peuvent être pensés séparément des processus de «frontiérisation externe ». Dans Europe, Constitution Frontière, Etienne Balibar propose ainsi une analytique de trois modèles dominants de «politisation de l'espace» (en lui en adjoignant un quatrième qui repose sur ses propres réflexions) ayant -ou ayant eu - un impact, non seulement sur les représentations des frontières internes et externes européennes, mais également sur les dynamiques sociales et politiques communément regroupées sous la désignation de mondialisation ${ }^{10}$. Si le contraste entre ces différents schémas n'est peut-être pas l'aspect le plus original de son travail, l'exposition des raisons pour lesquelles cette réflexion lui semble nécessaire donne à penser. Il s'agit d'organiser :

"la critique réfléchie des contradictions inhérentes à l'idée de “Constitution européenne” [...] Car si l'Europe aujourd'hui “triomphante” en pratique va aussi mal, et d'abord dans l'expérience de ses citoyens, il ne s'agit pas d'erreurs de tactique, de “mauvais choix" qu'au-

6. Ibid., p. 62.

7. Pour reprendre la terminologie de l'auteur. Voir en particulier: Balibar E., Europe Constitution Frontière, op. cit., pp. 76-91.

8. Ibid., p. 55. Soulignement d'origine.

9. Au sens, éventuellement, d'une désobéissance civique. Voir le chapitre éponyme dans: Balibar E., Droit de cité, op. cit., pp. 17-22. Il s'agit également, dans ce cadre, de soumettre « la question de savoir quelle constitution rédiger pour l'Union européenne ", qui a principalement occupé la convention constitutionnelle, à l'interrogation simultanée « de savoir ce qu'est une constitution, ou en quel sens une communauté politique, qu'elle soit médiate ou immédiate [...] a besoin d'un moment juridique pour définir son principe d'unité, conférer une valeur d'engagement (ou si l'on veut de fondation) à son surgissement et y inscrire un principe de durée ", Balibar E., Europe Constitution Frontière, op. cit., p. 55. Soulignement d'origine.

10. Le «choc des civilisations", le "réseau transnational mondial ", le «centre-périphérie » et pour le sien, les «nappes superposées ", Balibar E., Europe Constitution Frontière, op. cit., pp. 105-126. 
raient effectué les politiciens [...]. Il s'agit du fait que cette construction s'effectue dans un monde où les notions d'intérieur et d'extérieur ne sont et ne seront plus jamais strictement séparables, même sur le plan politique. Telle est la racine des "effets politiques pervers" qui transforment sous nos yeux les mesures de sécurité en facteurs d'insécurité croissante, ou l'attachement aux identités historiques en exil intérieur et en aliénation de masse au sein du monde contemporain ${ }^{11}{ }^{»}$.

En contradiction avec les « urgences de la conscience sociale » des peuples européens, les processus d'européanisation le sont donc également avec les processus dits de «mondialisation », position d'autant plus paradoxale que les premiers apparaissent à de nombreux égards parmi les précurseurs, parmi les produits les plus emblématiques des seconds. Il y a cependant ambiguïté, car la mise en contradiction des dynamiques d'européanisation et de mondialisation contemporaines pourrait ouvrir la voie à des modalités de « médiation » qu'un certain usage de l'Europe pourrait activer.

Cet usage de l'Europe "médiatrice » est au cour de l'ouvrage L'Europe, l'Amérique, la guerre. Il renvoie notamment à une certaine compréhension de la constitution historique longue de la notion d'Europe et de l'argumentaire selon lequel l'Europe en tant que telle n'a pas d'existence tranchée en dehors d'autres grands ensembles géo-politiques (atlantique, eurasien ou méditerranéen).

«S’il n’y a pas d'identité européenne opposable aux autres identités du monde, c'est parce qu'il n'y a pas de frontières absolues entre l'espace européen, historiquement et culturellement constitué, et les espaces qui l'enveloppent. Et s'il n'y a pas de frontières de l'Europe, c'est parce que l'Europe est elle-même une frontière [...] ou plus exactement une superposition de frontières, et donc de relations entre les histoires et les cultures du monde (ou du moins une grande partie d'entre elles), qu'elle réfléchit en son propre sein ${ }^{12}$. »

Il s'agirait alors de tirer toutes les leçons de l'histoire européenne, notamment celle des luttes politiques et sociales et de leur degré de reconnaissance institutionnelle, mais aussi des contradictions des processus d'européanisation et notamment des interstices ouverts par la remise en cause des identifications nationales et du monopole de la représentation démocratique dont bénéficient, aujourd'hui encore, les Etats nationaux de l'UE ${ }^{13}$, pour faire usage de

11. Ibid., pp. 153-154.

12. Balibar E., L'Europe, l'Amérique, la guerre, op. cit., p. 33.

13. Monopole dont la préservation est au cœur aujourd'hui des débats autour du traité de Lisbonne et de l'argumentaire en trompe-l'œil du président français notamment, selon lequel la légitimité de l'Union européenne passerait par l'efficacité dans la prise de décision et la mise en œuvre des politiques communes, discours de l'efficacité par consensus des gouvernants, 
l'Europe comme d'une modalité de transformation des dynamiques, notamment de violence, qui traversent l'espace des relations internationales contemporain. Cette médiation dite «évanouissante » par Balibar, regroupe finalement les éléments de sa réflexion présentés jusqu'ici :

«[...] d'une part que l'Europe, par constitution et par défaut de constitution, ne peut que se dérober à la mission dont elle-même ou d'autres voudraient la charger, ce qui est décevant; d'autre part que l'Europe pourrait contribuer de façon décisive, sinon à "transformer le monde" [...] du moins à en infléchir les évolutions annoncées, mais à la condition de "s'évanouir" à mesure que son intervention, ou sa médiation, se ferait plus déterminante : c'est-à-dire à la condition de se distinguer de plus en plus des images et des mythes de son "identité", enserrée par des frontières imaginaires, et de pénétrer de plus en plus profondément dans la logique des conflits qui déchirent le monde "commun" d'aujourd'hui, dont elle porte elle-même une part de la responsabilité historique ${ }^{14}$. »

Que retenir de ces éléments dans une perspective de recherche sur les processus d'européanisation? Force est de constater que travailler aujourd'hui sur l'Europe et ses frontières implique de se retrouver confronté à un affichage de l'urgence, à une conjonction de l'humanitaire et du sécuritaire et à une logique fondamentalement gestionnaire, c'est-à-dire préoccupée avant tout par l'efficacité ou, tout du moins, par sa mise en représentation. C'est assister finalement à une extension de la raison d'Etat national aux arènes gouvernementales communautaires et peut-être à l'émergence d'une raison d'Etat européenne ${ }^{15}$. En cela, les considérations développées par Etienne Balibar ne peuvent être d'application directe. Elles ont cependant le grand mérite de proposer des lignes de réflexion et des angles d'attaque qui permettent de ne pas se perdre dans les méandres d'une enquête strictement institutionnelle, de réintroduire une dimension (une conscience ?) politique dans les travaux sur l'Europe, et de décaler l'arrière-plan de telles recherches : non pas celui, forcément, des compétitions bureaucratiques sur fond de consensus politique mais celui, au contraire, des luttes politiques et sociales, des contournements et des désobéissances, qui constituent eux aussi un élément crucial et profondément ancré dans l'histoire européenne. L'ignorer ne reviendrait-il pas à occulter une opportunité de réinvention de l'espace des possibles pour une Europe qui se cherche toujours?

qui laisse bien peu de place aux possibilités de contestation et de reconduction des luttes politiques et sociales qui sont pourtant la condition fondamentale pour une Europe politique.

14 . Balibar E., L'Europe, l'Amérique, la guerre, op. cit., p. 53.

15. Ce qu'un collègue, dans ses travaux, a qualifié de "raison de la gouvernementalité européenne ». Voir Guittet E.-P., La Genèse de la coopération antiterroriste en Europe et l'implication de l'Espagne dans la (re)définition de l'identité européenne. De la raison d'Etat à la raison de la gouvernementalité européenne ?, thèse pour le doctorat de science politique, université Paris-X Nanterre, novembre 2006, 574 p. 